\title{
Why diachronically emergent properties must also be salient
}

\author{
Cyrille Imbert - Paris 1 / IHPST
}

Cyrille.Imbert@normalesup.org

To appear in "Philosophy and Complexity: Essays on Epistemology, Evolution, and Emergence", Edited by Carlos Gershenson, Diederik Aerts, and Bruce Edmonds, published by World Scientific.

\begin{abstract}
In this paper, I criticize Bedau's definition of 'diachronically emergent properties' (DEPs), which says that a property is a DEP if it can only be predicted by a simulation (simulation requirement) and is nominally emergent. I argue at length that this definition is not complete because it fails to eliminate trivial cases. I discuss the features that an additional criterion should meet in order to complete the definition and I develop a notion, salience, which together with the simulation requirement can be used to characterize DEPs. In the second part of the paper, I sketch this notion. Basically, a property is salient when one can find an indicator, namely a descriptive function (DF), that is such that its fitting description shifts from one elementary mathematical object (EMO) to another when the property appears. Finally, I discuss restrictions that must be brought to what can count as DFs and EMOs if the definition of salience is to work and be non trivial. I conclude that salience (or a refined version of it) can complete the definition of DEPs.
\end{abstract}

In the literature about emergent properties, attention has recently been paid to properties that emerge diachronically in complex systems, for example when a tornado ends up appearing in a usually quiet area.

The central idea behind diachronic emergence is that these emergent properties can be explained or derived, at least in principle, from the system microdynamics (so they have nothing mysterious), but only in a complex way, e.g. for the apparition of the tornado by numerically solving Navier-Stokes equations.

In the past decade, different definitions have been proposed to characterize more precisely diachronically emergent properties (DEPs). Stephan [9] emphasizes that DEPs are highly unpredictable, as demonstrated in the case of deterministic chaos. Bedau [1] claims that a property is diachronically emergent (he uses the term 'weakly emergent') if it can be derived from the system's microdynamics and external conditions but only by simulation. I label this latter condition the 'simulation requirement' (SR). 
In this paper, I focus on Bedau's definition, which I am definitely supportive of, and I try to refine it further by making it more stringent. I agree that the definition is sufficiently broad to be fruitfully applied to a large variety of systems that exhibit properties that seem to be DEPs. Yet, I argue that the SR is not enough to define DEPs and that the definition, as stated by Bedau, encompasses too many properties. I try in a second step to build a notion, namely salience, that is appropriate to complete the definition and capture only the right set of properties.

\section{Preliminaries}

In order to present the problem, I shall restrict for simplicity to an example belonging to discrete mathematics, namely the case of a one dimensional CA system composed of cells, each colored black or white. ${ }^{1}$ A rule determines the color of each cell at each step. Below is rule 110. It says for example that when a CA and its neighbours are black, the CA turns white at the next step.

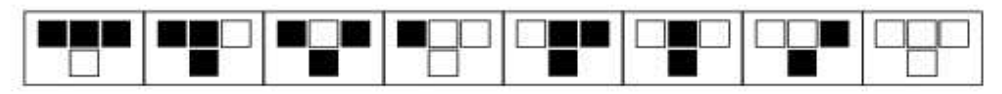

Figure 1: Rule 110

The evolution of such a CA system, composed of automata following rule 110, depends on its initial condition. Figures 2.1 and 2.2 represent this evolution when the initial condition is a single black cell. Each line represents the system state at a given step and the evolution is downwards.

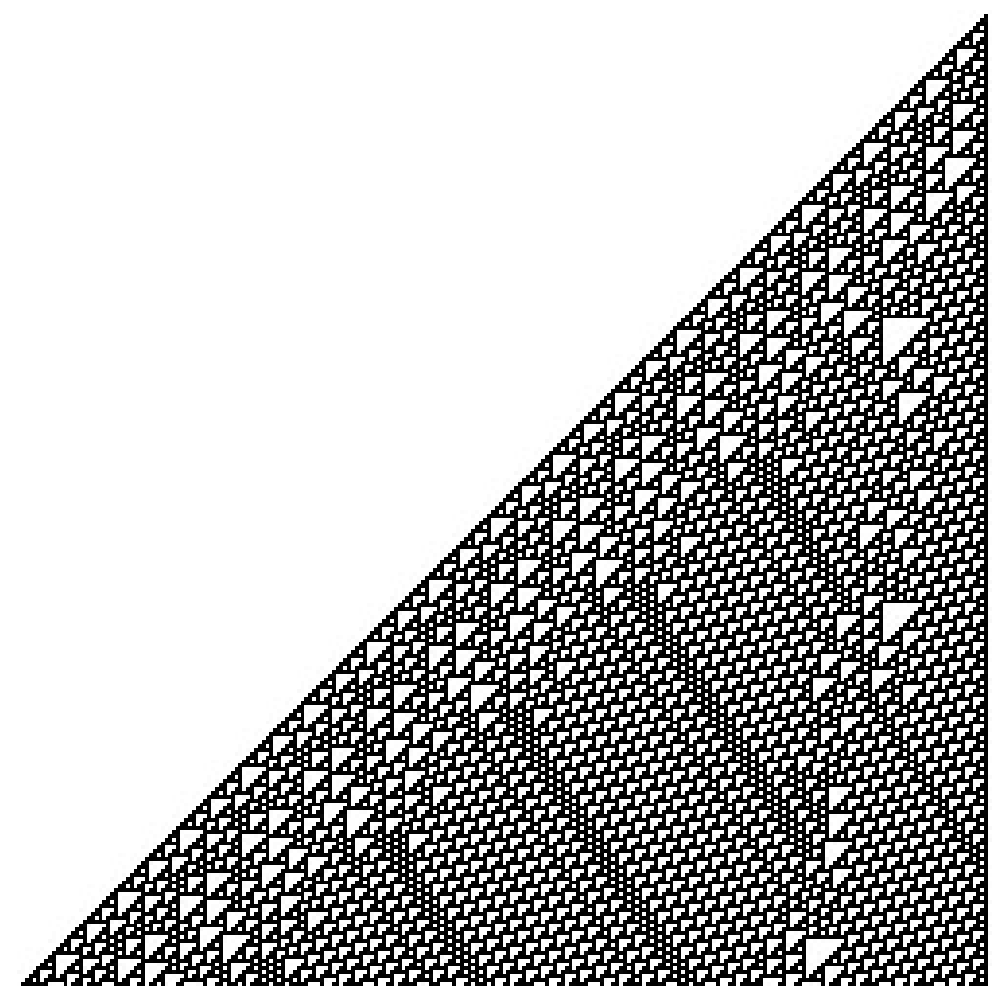

Figure 2.1: Rule 110 starting from a single black cell, 250 steps. Figure from $A$ New Kind of Science, chap. 2, reproduced with permission of Stephen Wolfram, LLC.

\footnotetext{
${ }^{1}$ For a clear presentation of cellular automata, see for example [10, chap. 2].
} 
One can see on these figures that in this case, the evolution is neither highly regular nor completely random. We can witness the emergence of patterns such as occasional big triangles or as the vertical column of triangles in the right.

For the discussion that follows, it is appropriate that I give pet names to the properties that the discussion is going to revolve upon. 'Blackcell' refers to the property 'having CA 10 in a black state' and it is true at time 20. 'Bigtriangle' refers to the property 'having a big triangle appearing around CA number 20 on the lattice' and it is true around time 80 . 'Microstate50' refers to the property 'being in the configuration that the system is in at step 50 ' and it is true at time 50. 'Verticalcolumn' refers to the property 'having a vertical column of triangles appearing around CA 60' and it is true around time 350. These are property names but for linguistic commodity, I shall also use these names to refer to the instantiation of these properties at the mentioned times and also as if they designated emerging things.

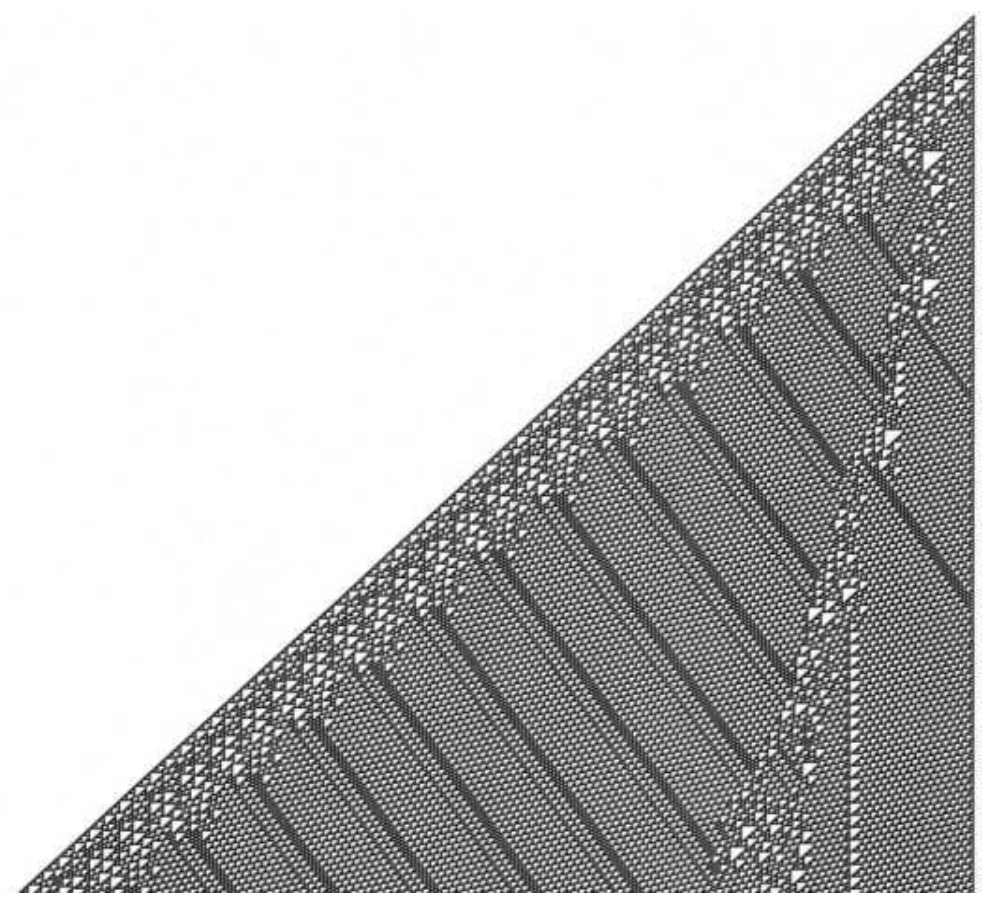

Figure 2.2 : Rule 110, 500 steps. Figure from A New Kind of Science, chap. 2, reproduced with permission of Stephen Wolfram, LLC.

I also need to introduce scraps of terminology. I shall use 'microproperty' to refer to a property of a basic constituent. In our examples, the two basic microproperties of a CA are 'being black' or 'being white' and you can specify them by including time, e.g. 'being black at time 3'. Following the uses in statistical physics, I shall call 'microstates' conjunctive properties that consist of the ordered enumeration of all the microproperties of the parts of the system $S$. I shall also call 'macroproperties' properties that can only be possessed by the whole system but not by its part. Such properties are sometimes referred to in the literature as 'nominally emergent' [2]. Structural properties, involving microproperties and a relation between them are a subspecies of nominally emergent properties [7]. Examples of macroproperties are Blackcell, Microstate50 or, for a cup of water, fluidity or transparency, because molecules of water cannot be said to be fluid or transparent. I will not venture here in giving a more detailed general definition of macroproperties because I shall not need that much to define DEPs. Bedau himself acknowledges that "full understanding of nominal 
emergence would require a general theory of when macro entities have a new kind of property that their constituents cannot have" [2]. That should not worry us too much because in our CA system example, we can very easily, if we need to, depart on a purely logical ground what counts as a macroproperty from what does not.

\section{Statement of the problem $(\mathrm{Pb})$}

I can now phrase precisely why I am not satisfied with Bedau's definition of DEPs. The consequence of equating the fact of being a DEP with the fact of satisfying the simulation requirement $(\mathrm{SR})$ is that

1. Blackcell, Microstate 50 and any coined conjunctive property that you feel like building up by the conjunction of microproperties, e.g. 'having CA 24 black at time 3 and CA 47 white at time 567 ' - I call these 'deceptive properties';

2. respectable properties such as Verticalcolumn, Bigtriangle, or any emerging pattern I call these "target properties";

must be treated on a par and considered as DEPs because we need to run a computer simulation to predict them all.

I think this is an unpalatable consequence of the definition, which shows that the SR is not enough to define DEPs.

\subsection{Two ways to face $(\mathrm{Pb})$}

I shall now examine two ways to answer my claim that the SR is not sufficient to define DEPs and I shall show why I think these answers are unsatisfactory.

\subsubsection{Is nominal emergence enough to solve $(\mathrm{Pb})$ ?}

I have assumed in my phrasing of the problem that the SR is what matters in the definition of DEPs. Bedau may perhaps argue that I have not faithfully reported his view, because he says: "Assume that $P$ is a nominally emergent property possessed by some locally reducible system $S$. Then $P$ is weakly emergent if and only if $P$ is derivable from all of $S$ 's micro facts but only by simulation". So according to Bedau the SR applies only to nominally emergent properties. Thus I can be reproached for having forgotten the condition about $P$ being nominally emergent or else for having misused the notion: Blackcell ('having CA 20 black') seems to be a property of the whole system and so to be nominally emergent... but isn't it because I used a logical trick to forge Blackcell, instead of simply talking of a less intricate microproperty which applies to CA 20 at time 10, namely 'being black'? Well, maybe. I leave to the reader's metaphysical intuitions whether the property 'having a big wart on her nose' (which is true of the witch that gives the apple to Snow White) is not a very respectable property too and is really the same as 'being in a warty state' (which, for a coarse-grained description of the witch, is true of the descriptive cell corresponding to the witch's nose). In any case, the conclusion should at least be that if we want to be able to determine which properties are DEPs we really need much more theorizing about nominal emergence to select the right properties.

But I strongly doubt that a good theory about nominal emergence will do. A reason is that, from a descriptive point of view, properties such as Bigtriangle or the appearance of a 
tornado in a fluid, which are paradigms for diachronical emergence, describe local features indeed, ${ }^{2}$ and that these features do not necessarily involve the whole system for being predicated but can be predicated of just a few CAs, even if they are generated by the whole system evolution (just like Blackcell). So from the nominal emergence perspective, the difference between Blackcell and Bigtriangle is very slight, whereas my guess is that Blackcell is not a DEP in our example, whereas the appearance of a tornado is.

Another problem is that properties which are pure conjunction of microproperties and involve the whole system, such as Microstate50, will still be nominally emergent, however unremarkable they may be. It is still possible to hope that a strengthening of nominal emergence may make it possible to get rid of them too. Yet it seems to me that with this strategy, we are progressively lead towards a different kind of emergence leaning on a strong distinction between whole and parts. The solution I propose in the second part of this paper, with the use of the notion of salience, does not make use at all of nominal emergence.

\subsubsection{Is there more to diachronical emergence than mere computational irre- ducibility?}

A second way to face $(\mathrm{Pb})$ is to hold tight like this: "Yes indeed, the SR is what only matters to define DEPs and there is no reason to make further distinctions between properties, because as far as weak emergence is concerned, these properties are really on a par. If you want to predict the apparition of Blackcell or Microstate50, you have to run a simulation and let all these properties diachronically emerge. These properties are so to speak "as diachronically emergent as" Bigtriangle or Verticalcolumn because the same computational effort (modulo time linearity to account for different appearance times) is required to predict them all. This computational effort corresponds precisely to the running of the dynamics of the whole system - and this is where the notion of whole system has a part to play."

I do not think that Bedau would hold tight like this. In [1], the examples are about the emergence of periodic or stable configurations in the Game of Life and about adaptation of mutation rates in a model of evolving life. That is not exactly what one would call trivial or insignificant properties of a system, as Blackcell or Microstate50 can be assessed to be. So if the definition purports to include in its scope such insignificant properties, this should be made clear and the examples are misleading because they point at a specific class of very remarkable DEPs.

Besides, if there is nothing more to diachronic emergence than the SR, then it is maybe better not to talk of emergence at all and to speak merely of properties of computationally irreducible systems [11, pp.737-750], or of properties that are for a system computationally irreducible: if one views the evolution of a system as a computation, then a system is computationally irreducible if given its initial state, there exists no shorter computation that enables to predict a later state of the system. In other words, there exists no shortcut to predict the properties of the system.

Yet, I am quite reluctant to content with an equivalence between diachronic emergence and computational irreducibility. Giving a clear definition of computational irreducibility and finding that there are systems that are computationally irreducible, as in the case of low-dimensional chaos [3], is one thing. Then, it could be a brute fact about nature that the properties that cannot be predicted except by a simulation are not really worthy of interest. Take for example a classical gas. Perhaps it is true that there is no way to predict exactly its future microstates (that is to say, to predict the position and velocity for each of the $10^{23}$

\footnotetext{
${ }^{2}$ See also note 7 .
} 
particles), except by a simulation. But who really cares? We can still use statistical physics to (try to) calculate more remarkable properties. So computational irreducibility would not be such a big issue, if it was just met in the case of such anonymous properties.

But this is not the case. And a good incentive to talk about diachronically emergent properties is the two-piece fact (S) that, as abundantly illustrated by [11] and as can be seen in figure 3 with Bigtriangle or Verticalcolumn:

(S1) there seems to be computationally irreducible systems and

(S2) these systems generate among other things some properties (e.g. periodicity when Verticalcolumn appears) that can be as respectable, remarkable, new, worthy of interest or what you like as the properties we ordinarily predict using analytical methods.

My conclusion is that there is really something wanting in a definition of diachronic emergence that only relies on the SR.

Various requirements can be found in the literature [8], for emergent properties, in particular novelty and irreducibility. ${ }^{3}$ The SR requirement seems to fulfill the irreducibility requirement. So my claim is that, in the case of diachronic emergence, something like the novelty requirement must also be honored one way or another.

\subsection{How to complete the simulation requirement?}

It did not go unnoticed that I left (S2) quite vague and used so far unsatisfactory adjectives ('interesting', 'remarkable', etc.). The purpose was to keep my different claims distinct so that one can agree with my diagnosis (the SR is not enough) and possibly disagree with the way I try to solve the problem (what is missing).

What I want to do now is to give a criterion that enables to depart the computationally irreducible properties that seem to have nothing special (deceptive properties) from the ones that seem to have something more (Verticalcolumn, Bigtriangle). But before presenting my solution, I think it is appropriate to list the constraints that weigh on this criterion.

\subsubsection{The criterion must not rely on intrinsic features of the property but must be contextual (C1)}

As I stated above, properties that are produced by a computationally irreducible system can be the same as the ones we predict in other analytically solvable cases, e.g. periodicity of a configuration. Therefore one should not be after a criterion that would be based on a logical analysis of each property, or on a complexity measure or any other feature characterizing intrinsically the properties. To put it differently, what is striking is not to witness a complex, or ordered or random behavior in a CA system but, for example, to witness the apparition of a complex or random behavior emerging out of an initial simple output or, like in the example of CA 110, of structures emerging out of an almost uniform initial condition. ${ }^{4}$ In other words, what matters is which new features the system dynamics is able to generate out of a context that did not possess these features, and that is why I say the criterion we look for is contextual.

\footnotetext{
${ }^{3}$ This is in no way is in a definition of or a necessary condition for emergent properties. In particularly, I do not assume that there is a single concept of emergence or that any concept of emergence must meet these requirements.

${ }^{4}$ See [11, pp. 149-152] for examples.
} 


\subsubsection{The criterion must be local, that is to say must rely on preceding mi- crostates only (C2)}

Most of the listed properties (deceptive and target properties) are new in a very weak sense: they are properties that were not possessed before (the system was never in a state identical to Microstate50 before). Verticalcolumn and Bigtriangle seem to be new in a stronger sense because the system seems to locally undergo a change. In the same time, nothing precludes that similar instances of target properties may have appeared before or may appear again later: tornadoes do re-emerge in the same areas. I conclude that the desired criterion, if it is to characterize novelty, must be local in the sense that it is possible to determine if a property is novel only by looking at the microstates of the system just before the property appears.

Local novelty is also an attractive feature because it is a weak requirement in contrast with other kinds of novelty such as absolute novelty (if the supposedly new features were never possessed before). And I am after a weak criterion because I believe that the SR is what matters first in diachronical emergence and I want only to refine it by disqualifying a very specific set of deceptive properties.

The requirement of local novelty is also there to do credit to the idea that the apparition of an emergent property corresponds to an observable change in the system state. Emergent properties may involve no new causal power, as this is precisely the case with diachronic emergence, where all causal effects can be traced back to the system's microdynamics. But one can still require that the emergence of these properties correspond to the apparition of novel features characterizing the system.

\subsubsection{The criterion must be contextually absolute (C3)}

Once a context - that is to say the previous microstates - is given, the novelty of a property must be assessed on the basis of objective properties. For example, there seems to be an objective sense in which one can say that a tornado is a new structure that locally appears, because one can find objective indicators to signal this appearance, such as a sudden local increase of average vorticity. In particular, although I call 'salience' the property that emergent properties must possess, I do not want salience to be relative to an observer and her cognitive apparatus.

\section{Salience}

In the last section of this paper, I try to develop a concept, salience, that fulfills the previously listed requirements and I claim that DEPs must also be salient. Salience is an independent notion and I believe that it can prove to have a wider application than the question of emergence. ${ }^{5}$ The definition of salience also raises its own set of problems, but since it is not my goal to fully develop this notion here, I shall be quite sketchy, leave aside most of these problems and be content to show how salience could be used to complete the definition of DEPs.

The idea I am going to develop is that a property is salient if one can find a descriptive indicator which can be calculated for any state of the system and that undergoes a change when a salient property appears. To make things clearer, I start with two clear-cut examples.

\footnotetext{
${ }^{5}$ For example for the question of what it is to identify a non trivial property in a data basis obtained by a number crunching simulation or by experiments.
} 
In the case of phase transitions, order parameters (when one manages to find one) yield such indicators. For example, in the case of the transition from ice to water, the quantity $\rho_{\text {ice }}-\rho_{\text {system }}$, where $\rho$ indicates the density, is zero till the transition and then grows.

Another more elaborate example from dynamical system field, which I borrow from Rueger[8], is the case of the damped non linear oscillator, which is described by van der Pol's equation: $\ddot{x}-a\left(1-x^{2}\right) \dot{x}+x=0$, where $\dot{x}=d x / d t$ and $a$ is a damping parameter. For a dynamical system, it is appropriate to study properties of the phase space, which plays for us the role of a descriptive indicator. With $a=0$, the oscillator is undamped and the phase space can be portrayed by concentric ellipses, each ellipse representing a trajectory (see figure 3.1). If the damping is gradually turned on, trajectories are no longer periodic and the phase space is made of spirales converging to a limit circle (see figure 3.2). So the turning off of the damping makes new salient properties appear (e.g. periodicity) and this can be indicated by the topological change of the phase space, which is described by topologically inequivalent objects (two objects are topologically inequivalent if one cannot smoothly deform one object to transform it into the other).

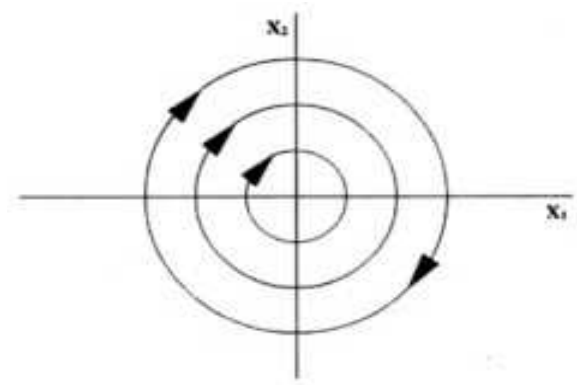

Figure 3.1: undamped oscillator, taken from Synthese, 124, 2004)

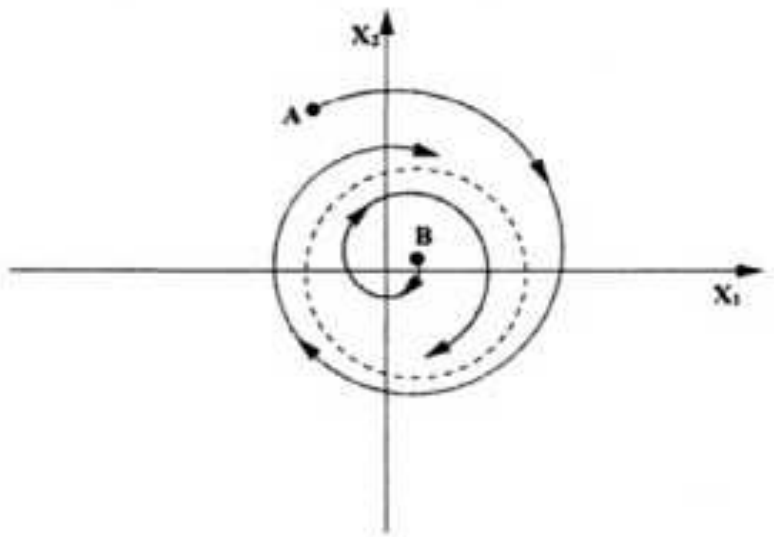

Figure 3.2 : damped oscillator, taken from Synthese, 124, 2004

This specific and very well chosen example enables Rueger to provide a very clear criterion of novelty, using the notion of topological inequivalence. Yet it seems to me that he actually shows how novelty can be defined in a very particular case (dynamical system studies) and not in general. I am not sure my criterion of salience will be as clear and elegant, but it aims at being more general. 


\subsection{Preliminary definitions}

I assume that at each time, the system can be suitably represented for the properties that are studied by data originating in $n$ measurements and I call 'state of the system' this n-tuple of measurements. Examples of states are a triplet $(\mathrm{P}, \mathrm{V}, \mathrm{T})$ for a gas at equilibrium, data describing a velocity field for a fluid, what I called 'microstates' for the above CA system or subparts of them (for example the subpart corresponding to CA number 15 to 25, that is to say where Bigtriangle does appear).

I call 'trajectory' the sequence of states that one gets by varying a parameter describing the system. This parameter can be time, like in the case of the above CA system, but it need not always be. For example, temperature can be chosen for an ice to water phase transition. What is only required to get a well-defined trajectory along a parameter is that one single state can be ascribed to each new value of the parameter along the trajectory. Suppose for example you study properties of cooling of glasses. Since, for a similar initial condition, the end state of the glass and its properties depend on the cooling rate (see figure 6), actual trajectories depend on the cooling rate. Therefore temperature cannot be taken as the varying parameter defining trajectories. Specifying exactly the trajectory does matter because salience is a contextual notion (C1) and because the part of the trajectory before a property appear provides the context against which the property stands out.

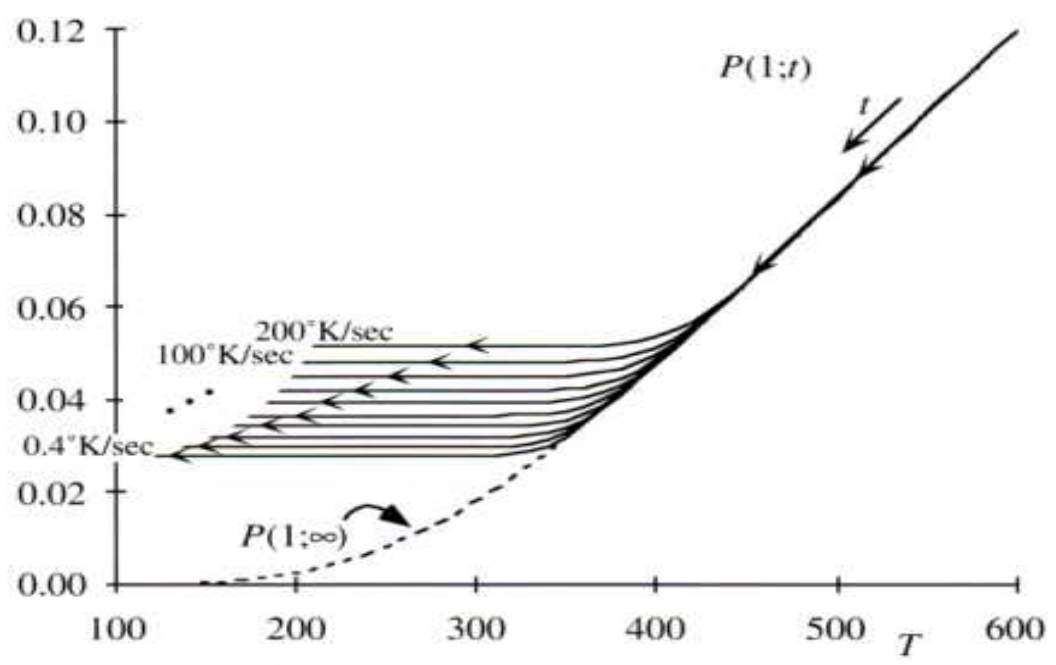

Figure 4: Glass cooling modelled by a two well system (the fraction of the systems in the higher well is plotted). Figure taken from Dynamics of Complex systems, p.107, by Bar-Yam, reproduced with permission of Addison-Wesley.

I call 'descriptive function' a single-valued mathematical function that takes as an argument the n-tuple describing the system. ${ }^{6}$

This is in a no way a definition of a new object. This definition only provides a label that is stamped on already existing functions. Here are examples of descriptive function:

\footnotetext{
${ }^{6}$ This is actually a restriction that I make for simplicity because one may need more complicate objects than single-valued function to describe suitably the systems. In Rueger's example of the oscillator, the system was described for each value of the damping parameter by trajectories within a phase space.
} 
- for a spin lattice, the total magnetization or typical size of clusters of similarly oriented spins;

- an estimation, using an auto-correlation function, of the correlation between measurements at spatial or temporal distance $r$ or $t$;

- the probability value of an event, for example of having two similar colors following each other on the above CA system;

- a complexity measure such as Kolmogorov complexity of a state;

- etc.

Descriptive functions are aimed at giving a common indicator all along the trajectory, so that it be possible to make comparisons and thus determine if the system exhibits a new behavior. In the case of Verticalcolumn, we can for example study the spatial correlations around CA number 60 in the 100 steps before Verticalcolumn is instantiated. On this interval, which plays the role of a context, the system undergoes fluctuations and correlations are low. When Verticalcolumn appears, fluctuations stop and the correlations suddenly get high.

To be more precise descriptive functions should themselves be described by other more elementary mathematical object (EMO) which enable to fit them locally. In the case of the oscillator, the phase space was described by different geometrical figures (circles and spirales). The novelty is then signaled by the shift in the description of the descriptive function from one EMO to another one. In the case of single-valued functions, EMOs are elementary functions such as $x^{2}$ and power laws, $\log (x), \exp (x)$, trigonometric functions, etc.

\subsection{Towards a definition of salience}

I can try now to propose a first definition of salience.

A property $P$ of a system $S$, which appears at $t$ or on interval $I$ is salient for trajectory $T$ of $S$ iff there exists different EMOs $A$ and $B$ and a descriptive function $D F$ of the states of $S$ 's trajectory such that:

- $D F$ is described by $A$ on an interval $C$ of $T$ (the context) before $t$ or $I$;

- the description of $D F$ shifts from $A$ to $B$ at $t$ or on $I$ with the apparition of property $P$.

This definition, because of the existence condition, does not provide an algorithm to determine if a property is salient. So the burden of the proof is for those who want to claim that a given property is salient, because they need to find an appropriate indicator, which can be far from obvious, even for phase transitions. Further, this definition, stated like this, can easily be found wanting. For example, it would be necessary to have a way to determine what the minimal size of the context needs to be in each case. In the following paragraphs, I shall study a few obvious corrections that should be brought to the definition in order to make it work better and also indicate in which directions some work needs to be done if a definition of this kind is to be given a chance to be well-grounded in the end. 


\subsection{How to avoid ad hoc descriptive functions?}

Remember salience aims at eliminating undesirable properties such as Microstate50 or Blackcell. An immediate objection is that it is very easy to find descriptive functions that will exhibit a change in behavior when Microstate50 or Blackcell appear. 'Blackcell' refers to the property of having CA 10 in a black state at time 20. If I take the color of CA 10 as a 1-tuple state, and if 'black' is encoded by 1 and 'white' by 0 , and if $C A(t, x)$ denotes the state of CA number $\mathrm{x}$ at time $t$, then the function $t \longmapsto \delta(t-20) . \delta(1-C A(t, 10))$, where $\delta$ denotes Dirac function, does exhibit a a sudden change in behavior when Blackcell appears. A similar trick can give the same result for Microstate50. That is why it is necessary to require that descriptive functions do not include functions of the parameter defining the trajectory (such as $t \longmapsto \delta(t-20))$ that have an ad hoc salient feature, which is made to appear at $t$ or $I$ (see definition above). Further, similar variables of the n-tuple should be treated similarly or contribute formally in a similar form to the descriptive function in order to avoid ad hoc treatments. The consequence is that most descriptive functions, like in the above examples, will be sums or integral functions.

A cautionary remark needs to be made here. Such restrictions are not aimed at getting rid of properties like Blackcell or Microstate50 in any circumstances. Suppose that CA 10 happens to be white from step 0 to 19 and then turns black at step 20. Then it is legitimate to say that Blackcell is salient and for that we no longer need an ad hoc function as above. Function $C A(t, 10)$, which is tolerated by the definition, will nicely do the job because it will have a zero value till step 19 and then will abruptly take value 1 at step $20{ }^{7}$ Similarly, if it happens that Microstate50 is random and appears after a succession of non-random states, then this provides a good ground to call it 'salient'.

\subsection{Requirements about describing EMOs}

Notice first that I do not define in the abstract what can count as EMOs. What EMOs are will depend in each case of what kind of mathematical object the descriptive function is. For example, EMOs will be different if you want to study the evolution of a phase space or of an order parameter.

A fully assumed consequence of my definition is that a curve can have different salient parts even if you can fit it with one single curve. Take for example a curve that you can fit with function $x \longmapsto x^{2}+0.1 x^{3}$. With my definition, it can be said to have different salient parts. Around $x=0$, the curve is correctly described by $x^{2}$. For large $\mathrm{x}$, it is best described by $0.1 x^{3}$.

A more worrisome problem is this. Take a curve $F$ that always has 0 value. Then you can very easily find two polynomials $A$ and $B$ such that 1 ) $A$ has value 0 till $\mathrm{x}=1$ and then has a more complicated behavior 2) $B$ has value 0 after $x=1$ and a complicated behavior before. ${ }^{8}$ $A$ and $B$ are 2 EMOs and the description of $F$ shifts from one to the other. So it seems that it is possible to find salience really everywhere. To guard against that, it is necessary to require that there is no EMO that fits $F$ on both intervals where $A$ and $B$ are supposed

\footnotetext{
${ }^{7}$ Note that in this case, nominal emergence would disqualify Blackcell.

${ }^{8}$ In short, I need to set restrictions on which EMOs can be used to fit the curves. It probably did not go unnoticed to the reader that the discussion of salience turns out to be reminiscent of discussions about induction, curve-fitting and projectibility, and here in particular of Goodman's paradox about grue emeralds (see [5, chap. 3-4] and [4, chap. 5]). A complete definition of salience should show how to avoid the goodmanian pitfalls or how to get out of them.
} 
to fit it and that is in the same time simpler than $A$ and $B$. Here, the function $x \longmapsto 0$ is simpler than $A$ and $B$.

The outcome of this is that, in order to fully develop the notion of salience, I need to be able to estimate the simplicity of mathematical objects. In the previous example, one must be able to explain why $x^{2}+0.1 x^{3}$ is less simple than $x^{2}$ or $0.1 x^{3}$. Since the last two expressions are composed of one element and the former of these two elements, the answer may seem obvious. But this seems all too relative to the mathematical framework that is taken as basic. Taking as basic the functions $x \longmapsto g(x)=x^{2}+0.1 x^{3}$ and $x \longmapsto h(x)=x^{2}-0.1 x^{3}$, one gets $x^{2}=0.5(g(x)+h(x))$ and $x^{2}$ seems now less simple than $g(x)$. As a reviewer points out, this makes salience relative to a privileged descriptive mathematical framework. A way out of the deadlock would perhaps be to argue that there are good objective grounds, in each case of data mining and salient properties detection, to privilege a descriptive mathematical framework and basic EMOs on the basis of which projectable quantities characterizing the system the descriptive function and more generally the mathematical description represent.

I do not go any further in the discussion of the concept of salience, since as just shown, this would require a close scrutiny of much debated questions, like simplicity and curve fitting. One more thing still. In this paper, the concept of salience was only aimed at giving a way to single out the properties that are considered as remarkable in the study of a system, e.g. in physics. But determining what patterns or properties are salient for a subject is also a question that is crucial in cognitive sciences. ${ }^{9}$ I make no claim in this paper about the link between the two notions, which may be somewhat related, even if not identical. This latter point can be illustrated as follows. Indeed, a salient property (as defined above) in a data basis can be imperceptible for a subject. That is why data mining requires resort to statistical tests made by computers. In the same time, perceptual abilities for pattern recognition also prove very useful to detect interesting properties, for example in hydrodynamic simulations, and the existence of the supposedly detected properties can be checked afterwards with statistical indicators. Finally, a system (e.g. a Hopfield network [10]) could be trained to detect in any circumstances a given pattern of CAs whereas this pattern need not always be salient (in my sense), since the notion is contextual. Besides, the pattern detection system may treat differently inputs of the same kind (i.e. corresponding to similar variables of the n-tuple in the mathematical description) and thereby use for detection a function that would not qualify as a descriptive function.

\section{Conclusion}

We can now check that the concept of salience meets the requirements listed above, or that a well-grounded and refined version of it could be hoped to. It is local, because it depends only on the preceding part of the trajectory. It is contextual because any property can happen to be salient if it is generated by a context against which some of its features stand out. It is objective (at least provided a descriptive mathematical framework is given) because it relies on the construction of mathematical indicators and not on our epistemic interests, perceptual abilities or practical goals: for example, the position at a given time of a planet, on which one wants to send a shuttle, is very unlikely to be salient.

Salience, as I built it, is a very weak and purely descriptive notion, which aims at grasping the idea that the apparition of a non-trivial property is simultaneous with a significant change in some of the values of the observable quantities characterizing the system. Once again,

\footnotetext{
${ }^{9}$ See [6] for example.
} 
I think that using a concept as weak as possible to complete the definition of DEPs is appropriate because I believe that the SR is the crucial element in it. But my conclusion is that DEPs satisfy the SR and are salient.

\section{Acknowledgements}

Special thanks to Anouk Barberousse, Jacques Dubucs and Paul Humphreys for discussing different versions of this paper and also to an anonymous reviewer for very helpful and very apposite critical comments, which helped a lot to improve the paper. All remaining shortcomings are of course mine.

\section{References}

[1] Mark Bedau. Weak emergence. Philosophical Perspectives : Mind, Causation and Word, 11, 1997.

[2] Mark Bedau. Downward causation and the autonomy of weak emergence. Principia, 6, 2003.

[3] J.P Crutchfield, J.D Farmer, N.H Packard, and R.S Shaw. Chaos. Scientific American, $255,1986$.

[4] Nelson Goodman. Language of Arts. Hackett Publishing Company,Inc., 1976.

[5] Nelson Goodman. Fact Fiction, and Forecast. Harvard University Press, 1983.

[6] David Marr. Vision: a computational investigation into the human representation and processing of visual information. New York: Freeman, 1982.

[7] Timothy O'Connor and Hong Yu Wong. The metaphysics of emergence. Nô̂s. forthcoming.

[8] Alex Rueger. Physical emergence, diachronic and synchronic. Synthese, 124, 2000.

[9] Achim Stephan. Phenomenal emergence. Networks, 3-4, 2004.

[10] Gérard Weisbuch. Dynamique des systèmes complexes, Une introduction aux réseaux d'automates. InterEditions/Editions du CNRS, 1989.

[11] Stephen Wolfram. A New Kind of Science. Wolfram Media, Inc., 2002. 